OPEN ACCESS

Edited by:

Ru-Ping Dai,

Central South University, China

Reviewed by:

Zhengyuan Xia,

The University of Hong Kong,

Hong Kong

Zhaolan $\mathrm{Hu}$

Stanford University, United States

*Correspondence:

You Shang

you_shanghust@163.com

Shiying Yuan

shiying_yuan@163.com

Dingyu Zhang

1813886398@qq.com

Dechang Chen

icudechangchen@163.com

tThese authors have contributed equally to this work

Specialty section: This article was submitted to Intensive Care Medicine and Anesthesiology,

a section of the journal

Frontiers in Medicine

Received: 18 September 2020 Accepted: 10 November 2020 Published: 08 December 2020

Citation:

Xu J, Yang $X$, Huang $C$, Zou $X$, Zhou $T$

Pan S, Yang $L, W u Y$, Ouyang $Y$, Wang $Y$, Xu D, Zhao $X$, Shu $H$, Jiang $Y$, Xiong W, Ren L, Liu H, Yuan Y, Qi H,

Fu S, Chen D, Zhang D, Yuan S and

Shang Y (2020) A Novel

Risk-Stratification Models of the High-Flow Nasal Cannula Therapy in COVID-19 Patients With Hypoxemic

Respiratory Failure.

Front. Med. 7:607821.

doi: 10.3389/fmed.2020.607821

\section{A Novel Risk-Stratification Models of the High-Flow Nasal Cannula Therapy in COVID-19 Patients With Hypoxemic Respiratory Failure}

\author{
Jiqian $\mathrm{Xu}^{1+}$, Xiaobo Yang ${ }^{1+}$, Chaolin Huang ${ }^{2 \dagger}$, Xiaojing Zou ${ }^{1+}$, Ting Zhou ${ }^{1+}$, \\ Shangwen Pan ${ }^{1+}$, Luyu Yang ${ }^{3}$, Yongran Wu ${ }^{1}$, Yaqi Ouyang ${ }^{1}$, Yaxin Wang ${ }^{1}$, Dan $\mathrm{Xu}^{1}$, \\ Xin Zhao ${ }^{1}$, Huaqing Shu ${ }^{1}$, Yongxiang Jiang ${ }^{1}$, Wei Xiong ${ }^{1}$, Lehao Ren ${ }^{1}$, Hong Liu ${ }^{1,2}$, \\ Yin Yuan ${ }^{1}$, Hong $Q i^{1}$, Shouzhi Fu ${ }^{3}$, Dechang Chen ${ }^{4 *}$, Dingyu Zhang ${ }^{2 *}$, Shiying Yuan ${ }^{1 *}$ and \\ You Shang $1,2,5 *$
}

${ }^{1}$ Department of Critical Care Medicine, Union Hospital, Tongji Medical College, Huazhong University of Science and Technology, Wuhan, China, ${ }^{2}$ Research Center for Translational Medicine, Jinyintan Hospital, Wuhan, China, ${ }^{3}$ Department of ICU/Emergency Wuhan Third Hospital, Wuhan University, Wuhan, China, ${ }^{4}$ Department of Pulmonary and Critical Care Medicine, Ruijin Hospital, Shanghai Jiao Tong University School of Medicine, Shanghai, China, ${ }^{5}$ Institute of Anesthesiology and Critical Care Medicine, Union Hospital, Tongji Medical College, Huazhong University of Science and Technology, Wuhan, China

Background: High-flow nasal cannula (HFNC) has been recommended as a suitable choice for the management of coronavirus disease 2019 (COVID-19) patients with acute hypoxemic respiratory failure before mechanical ventilation (MV); however, delaying MV with HFNC therapy is still a dilemma between the technique and clinical management during the ongoing pandemic.

Methods: Retrospective analysis of COVID-19 patients treated with HFNC therapy from four hospitals of Wuhan, China. Demographic information and clinical variables before, at, and shortly after HFNC initiation were collected and analyzed. A risk-stratification model of HFNC failure (the need for MV) was developed with the 324 patients of Jin Yin-tan Hospital and validated its accuracy with 69 patients of other hospitals.

Results: Among the training cohort, the median duration of HFNC therapy was 6 (range, 3-11), and 147 experienced HFNC failure within 7 days of HFNC initiation. Early predictors of HFNC failure on the basis of a multivariate regression analysis included age older than 60 years [odds ratio (OR),1.93; 95\% confidence interval (Cl), 1.08-3.44; $p=0.027 ; 2$ points], respiratory rate-oxygenation index (ROX) $<5.31$ (OR, 5.22; 95\% $\mathrm{Cl}, 2.96-9.20 ; p<0.001 ; 5$ points) within the first $4 \mathrm{~h}$ of HFNC initiation, platelets $<125 \times 10^{9} / \mathrm{L}(\mathrm{OR}, 3.04 ; 95 \% \mathrm{Cl}, 1.46-6.35 ; p=0.003 ; 3$ points), and interleukin 6 (IL-6) >7.0 pg/mL (OR, 3.34; 95\% Cl, 1.79-6.23; $p<0.001 ; 3$ points) at HFNC initiation. A weighted risk-stratification model of these predictors showed sensitivity of $80.3 \%$, specificity of $71.2 \%$ and a better predictive ability than ROX index alone [area under the curve $(A \cup C)=0.807$ vs. 0.779, $p<0.001$. Six points were used as a cutoff value for the risk of HFNC failure stratification. The HFNC success probability of patients in low-risk group (84.2\%) was 9.84 times that in the high-risk group (34.8\%). In the subsequent validation cohort, the AUC of the model was $0.815(0.71-0.92)$. 
Conclusions: Aged patients with lower ROX index, thrombocytopenia, and elevated IL-6 values are at increased risk of HFNC failure. The risk-stratification models accurately predicted the HFNC failure and early stratified COVID-19 patients with HFNC therapy into relevant risk categories.

Keywords: COVID-19, HFNC, ROX, mechanical ventilation, thrombocytopenia, risk-stratification

\section{INTRODUCTION}

Severe coronavirus disease 2019 (COVID-19) caused by severe acute respiratory syndrome coronavirus 2 (SARS-CoV2) infection was characterized by progressive dyspnea and hypoxemia within 1 week after onset of the disease (1-4). As of August 23, 2020, more than 1.7 million new COVID-19 patients were reported in the last 7 days, by the World Health Organization Region. For most patients, they recovered on conventional oxygen therapy. However, some patients, mainly critically ill patients, progressed to severe respiratory distress, and needed advanced oxygen therapy, including high-flow nasal cannula (HFNC) therapy and mechanical ventilation (MV) (2).

HFNC can bring high concentrations of humidified oxygen with low level of positive end-expiratory pressure; facilitate the elimination of carbon dioxide, allowing one to rapidly relieve the acute hypoxemic respiratory failure (AHRF) symptoms $(5,6)$; and apply in critical care resource-limited countries. During the ongoing pandemic, HFNC has been recommended as a bridge for management of patients with severe or critical COVID-19 on the basis of the evidences from non-coronavirus-related pneumonia (6-9). To our best knowledge, however, one important concern during HFNC therapy in non-COVID-19 patients with AHRF is to not delay the need of MV (10), and the association between HFNC therapy and its outcome (need or not for MV) and its early predictors has not been explored.

Here, we reported the clinical course of COVID-19 patients receiving HFNC and explored the predictors and attempted to develop a novel risk-stratification model that predicts the failure (the need for MV) of HFNC therapy for COVID-19 patients and early stratifies them into relevant risk categories.

\section{METHODS}

\section{Study Design and Participants}

This was a multicenter retrospective observational study on adult COVID-19 patients receiving HFNC oxygen therapy in Union Hospital, Wuhan Third Hospital, Union Hospital West Campus, or Jinyintan Hospital between December 29, 2019, and April 30, 2020. The training cohort was set up in Jin Yin-tan Hospital, and the validation cohort was set up in the other hospitals. All patients with a diagnosis of COVID-19 according to guidelines released by the National Health Commission of the People's Republic of China were screened (11). Patients were excluded if they never received HFNC, received HFNC after MV, deceased within $12 \mathrm{~h}$ after admission, had important information that was missing within $12 \mathrm{~h}$ of HFNC therapy, or were included in previous studies $(2,4,12,13)$. Research approval (2020-0041-1) was granted by the ethics board of Wuhan Union Hospital as the central coordinating center. The need for informed consent was waived.

\section{HFNC Device and Treatment}

HFNC was considered if a patient with COVID-19 required a conventional oxygen therapy with oxygen flow higher than 10 $\mathrm{L} / \mathrm{min}$ to achieve $\mathrm{SpO}_{2}$ of $>90 \%$ or a respiratory rate $(\mathrm{RR})>30$ breaths per $\min (\mathrm{bpm})$ despite adequate oxygen supplementation or showed persistent signs of respiratory distress (10, 14-17).

The included COVID-19 patients received HFNC oxygen therapy via HFNC device (Fisher \& Paykel, Auckland, New Zealand). The temperature of the heated humidification system was adjusted between 31 and $37^{\circ} \mathrm{C}$ to provide optimal humidity; the flow was initiated with a minimum flow at $30 \mathrm{~L} / \mathrm{min}$, and $\mathrm{FIO}_{2}$ was adjusted to reach $\mathrm{SpO}_{2}>90 \%$ or higher $(10,14,18,19)$.

The discontinuation of HFNC oxygen therapy and the switch to either conventional oxygen therapy or MV, was made at the discretion of the treating physicians. If respiratory failure improved, a trial of intermittent HFNC therapy was performed, and the duration of HFNC was gradually shortened until the HFNC was totally substituted by conventional oxygen therapy. If the respiratory failure progressively deteriorated, non-invasive or invasive MV was used. The decision to intubate was at the discretion of the treating physicians in accordance with published guidelines [26].

\section{Data Collection}

Patient identification in these ICUs was achieved by reviewing admission logs from available medical records. After several cycles of feedback and pilot testing, modified standardized International Severe Acute Respiratory and Emerging Infection Consortium case report forms for COVID-19 were utilized (12). Data were extracted from local servers by experienced research physicians at each center.

Demographic data, preexisting comorbidities (chronic cardiac disease, chronic pulmonary disease, hypertension, cerebrovascular disease, chronic liver disease, diabetes, and malignancy), vital signs ( $R R$, heart rate, blood pressure), and laboratory values [white blood cell count, platelets, prothrombin time (PT), activated partial thromboplastin time (APTT), plasma D-dimer, plasma fibrinogen, alanine aminotransferase (ALT), aspartate aminotransferase (AST), serum creatinine, hypersensitive troponin I (hsTNI), IL-6, arterial blood gas analysis], complications [acute cardiac injury, acute kidney injury (AKI), liver dysfunction, coagulopathy, and hospital acquired infection], and treatments (oxygen therapy, corticosteroids) were collected at admission and HFNC and MV initiation. 


\section{Definitions}

AKI was diagnosed based on the serum creatinine criterion of KDIGO (20). Acute cardiac injury was diagnosed if the serum concentration of hsTNI was measured in the laboratory above the upper limit of the reference range ( $>28 \mathrm{pg} / \mathrm{mL})$ (12). Liver dysfunction was diagnosed if serum ALT $>50 \mathrm{U} / \mathrm{L}$ or AST $>40$ U/L (21). Coagulopathy was defined if PT $>16.5 \mathrm{~s}$ or APTT $>42 \mathrm{~s}$ (13). Shock was defined according to the 2016 Third International Consensus Definition for Sepsis and Septic Shock (22). Respiratory rate-oxygenation (ROX) was defined as the ratio of $\mathrm{SpO}_{2} / \mathrm{FIO}_{2}$ to respiratory rate $(18,23)$. HFNC therapy failure was defined as need for MV within 7 days of HFNC initiation (24). The criteria for typical HFNC failures include failures to maintain $\mathrm{SpO}_{2}>90 \%$, hypercapnic respiratory failures with $\mathrm{pH}<7.2$ or $\mathrm{PaCO}_{2}$ of arterial blood $>55 \mathrm{~mm} \mathrm{Hg}$, worsening respiratory distress, or need for airway protection due to altered mental state or aspiration $(10,14,15,18,19)$.

\section{Statistical Analysis}

No hypothesis was made for the present study, so sample size estimation was unavailable. Data were expressed as mean \pm standard deviation, median [interquartile range (IQR)] or median (range) for continuous variables, and number (\%) for categorical variables. Differences between patients with failure and success of HFNC oxygen therapy, and between survivors and non-survivors of MV after HFNC failure, were explored using two-sample $t$-test for parametric variables, Wilcoxon ranksum test for non-parametric variables, and Fisher exact test for categorical variables. Age was dichotomized at 60 years (25). IL6 , D-dimer, and platelet count were dichotomized at the clinically relevant cutoff. ROX index was also included and dichotomized at the optimal cutoff point following Youden index of receiver operating characteristic (ROC) curve (18). Dichotomized age, IL-6, platelet counts, comorbidities, and complications showing a $p<0.1$ in univariate analysis were included for multiple logistic regression analysis. The scores were assigned as integer values relative to the regression coefficient of each predictor. The predictive value of the risk-stratification models was assessed by the area under the curve (AUC). Cutoff points were calculated according to Youden index of ROC. Success curves of HFNC oxygen therapy between low-risk and high-risk of HFNC failure following the cutoff value and survival analysis for the patients who received MV before and after $48 \mathrm{~h}$ of HFNC initiation were developed using the Kaplan-Meier method.

All statistical tests were 2 -tailed with significance set at $p<$ 0.05. The Stata/IC 15.1 software (StataCorp, College Station, TX, USA) was applied for all analyses.

\section{RESULTS}

\section{Demographic Data and Clinical Parameters of Included Patients}

We screened 3,102 patients with confirmed COVID-19 between December 29, 2019, and March 30, 2020, of which 546 patients (17.6\%) with severe AHRF were identified, and 324 patients were included into training cohort, and 69 patients formed the validation cohort.
Among the training cohort, the mean age of COVID-19 patients was $63.2 \pm 14.5$ years, of whom 211 patients $(65.1 \%)$ were older than 60 years, and $219(67.6 \%)$ were male (Table 1 and Supplementary Table 1 ). The proportion of patients with coexisting conditions were not significantly different between the HFNC success group and the HFNC failure group except for malignancy ( $p=0.024)$. The ratio of positive SARS-CoV-2 RNA at HFNC initiation and the duration of HFNC initiation to the negative conversion of SARS-CoV-2 RNA for the survivors was not different between the two groups.

At HFNC initiation, compared with patients in the HFNC success group, the HFNC failure group had a higher level of D-dimer [4.8 (1.1-17.7) vs. $2.6(0.8-8.8) ; p=0.0203$ ] and more patients with IL-6 $>7.0 \mathrm{pg} / \mathrm{mL}[124(84.4 \%)$ vs. 99 (55.9\%); $p<0.001]$ and with platelets $<125 \times 10^{9} / \mathrm{L}$ [22 (12.4\%) vs. $43(29.3 \%), p<0.001]$, but no differences in eosinophils, lymphocytes, the proportion of elevated Ddimer, and concentration of plasma fibrinogen and IL-6 were observed between the two groups. Moreover, there was a higher prevalence of AKI [26 (17.7\%) vs. $11(6.2 \%) ; p=0.001]$ and acute cardiac injury [97 (66.0\%) vs. 91 (51.4\%); $p=0.006$ ] in the HFNC failure group, but no differences in shock, liver dysfunction, coagulopathy, and corticosteroids treatment were observed between the two groups.

\section{Respiratory Variables During HFNC Treatment of the Training Cohort}

At baseline, 218 patients had analyses of arterial blood gas, and the number of patients with $\mathrm{PaO}_{2} / \mathrm{FIO}_{2}<200 \mathrm{~mm} \mathrm{Hg}$ was 174. The patients in the HFNC failure group had lower levels of $\mathrm{PaO}_{2} / \mathrm{FIO}_{2}[129.6(100.0-163.4)$ vs. 151.2 (122.8-199.2) mm Hg; $p<0.001]$.

After HFNC therapy, the patients in the HFNC success group had higher $\mathrm{SpO}_{2} / \mathrm{FIO}_{2}[134.2$ (117.5-158.3) vs. 108.7 (94.6-116.3) fold; $p<0.001$ ] and lower RR [22 (20-24) vs. 23 (22-25); $p<$ 0.001 ] (Table 2), but no differences in systolic arterial pressure were observed between the two groups. The RR alone had an AUC of 0.65 (0.59-0.71). Higher ROX index values with an AUC of $0.779(0.73-0.83)$ were observed in the HFNC success group [5.9 (5.0-7.5) vs. $4.8(4.1-5.3) ; p<0.001]$. The optimal cutoff point for the ROX index within the first $4 \mathrm{~h}$ was estimated to be 5.31 in accordance to the ROC curve, more patients in the HFNC failure group had an ROX index score $<5.31$ [114 (77.6\%) vs. 60 (33.9\%), $p<0.001]$.

\section{The Outcomes of HFNC Therapy of the Training Cohort and Its Predictors}

The median duration of HFNC therapy was 6 (range, 3-11). One hundred forty-seven (46.4\%) experienced HFNC failure within 7 days of HFNC initiation.

Multiple logistic regression analysis revealed the independent predictors of HFNC failure (Figure 1), comprising age older than 60 years [odds ratio (OR) 2.54; 95\% CI, 1.39-4.65; $p=0.003$ ], platelets $<125 \times 10^{9} / \mathrm{L}(\mathrm{OR}, 3.18$; 95\% CI, 1.36-7.46; $p=0.008$ ), and IL-6 $>7.0 \mathrm{pg} / \mathrm{mL}$ (OR, 3.07; 95\% CI, 1.67-5.62; $p<0.001 ;$ ) at 
TABLE 1 | Baseline characteristics of the included 324 patients with COVID-19 at HFNC initiation.

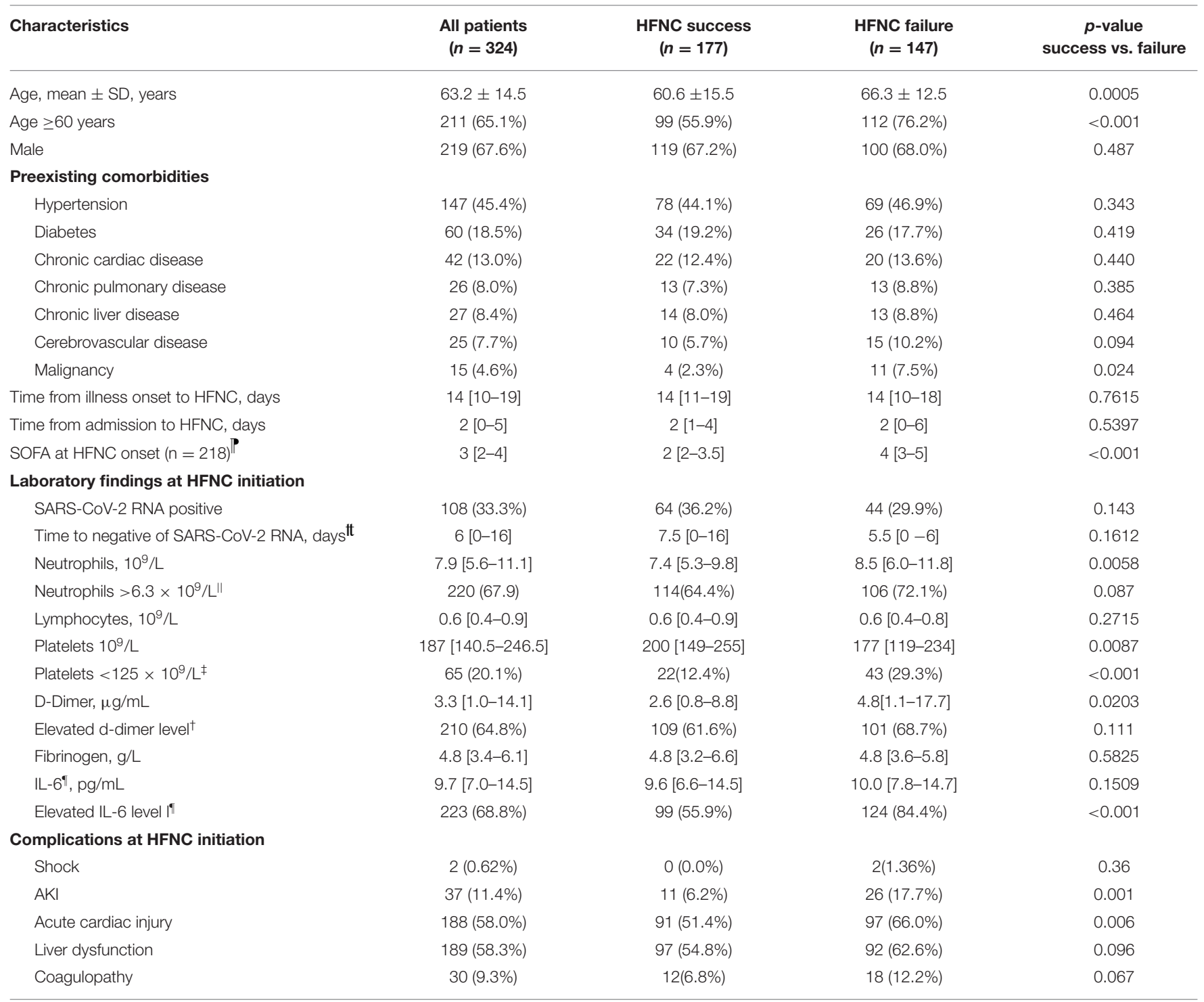

AKI, acute kidney injury; COVID-19, coronavirus disease 2019; IQR, interquartile range; SD, standard deviation; HFNC, high-flow nasal cannula; IL-6, interleukin 6; SOFA, Sequential Organ Failure Assessment.

Data were expressed median [interquartile range] or as mean \pm standard deviation.

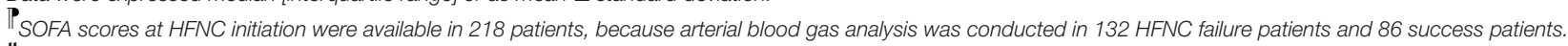

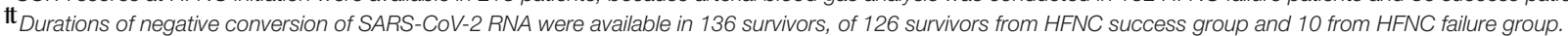

"The upper limit of normal range of neutrophil count was $6.3 \times 10^{9} / \mathrm{L}$.

t The upper limit of normal range of d-dimer was $1.5 \mu \mathrm{g} / \mathrm{mL}$.

${ }^{\ddagger}$ The lower limit of normal range of platelet count was $125 \times 10^{9} / \mathrm{L}$.

IT The upper limit of normal range was $7 \mathrm{pg} / \mathrm{mL}$.

HFNC initiation, ROX index $<5.5$ (OR, 5.92; 95\% CI, 3.31-10.58, $p<0.001$ ) within the first $4 \mathrm{~h}$ of HFNC therapy.

\section{Risk-Stratification Models of HFNC Therapy in COVID-19 Patients}

A special risk-stratification model with relative weights according to the regression coefficient of each independent predictors is shown in Figure 1. Patients with older than 60 years were assigned a score of 2; platelets $>125 \times 10^{9} / \mathrm{L}$ and IL- $6>0.7$ $\mathrm{pg} / \mathrm{mL}$ were given a score of 3 ; ROX index $<5.31$ was assigned a score of 5 .

The models had an AUC of 0.807 (0.76-0.85), 80.3\% sensitivity, $71.2 \%$ specificity, and a greater accuracy than the ROX index alone (AUC $=0.779$ ) in predicting the HFNC failure for COVID-19 patients (Figure 2A). The cutoff value of the models for the risk of HFNC failure stratification was six points.

For all patients allocated into training cohort, 55 patients were deceased on HFNC therapy due to do-not-intubate (excluded 
TABLE 2 | Physiologic variables at HFNC initiation and $12 \mathrm{~h}$ of HFNC onset.

\begin{tabular}{|c|c|c|c|c|}
\hline Parameters & $\begin{array}{l}\text { All patients } \\
(n=324)\end{array}$ & $\begin{array}{l}\text { HFNC success } \\
\quad(n=177)\end{array}$ & $\begin{array}{l}\text { HFNC failure } \\
\quad(n=147)\end{array}$ & $\begin{array}{c}p \text {-value } \\
\text { success vs. } \\
\text { failure }\end{array}$ \\
\hline $\begin{array}{l}\text { Heart rate at HFNC } \\
\text { initiation }\end{array}$ & $88.8 \pm 16.3$ & $87.0 \pm 13.8$ & $91.0 \pm 18.7$ & 0.0121 \\
\hline $\begin{array}{l}\text { Systolic arterial pressure } \\
\text { at HFNC initiation }\end{array}$ & $129.2 \pm 17.4$ & $128.3 \pm 17.0$ & $130.3 \pm 17.8$ & 0.1612 \\
\hline \multicolumn{5}{|c|}{ Arterial blood gas analysis at HFNC initiation $\mathbb{P}$} \\
\hline $\mathrm{pH}$ & $7.47 \pm 0.05$ & $7.46 \pm 0.04$ & $7.47 \pm 0.07$ & 0.1103 \\
\hline $\mathrm{PaCO}_{2}, \mathrm{~mm} \mathrm{Hg}$ & $34.3 \pm 7.7$ & $34.9 \pm 6.5$ & $33.5 \pm 9.1$ & 0.0106 \\
\hline $\mathrm{HCO}_{3}^{-}, \mathrm{mmol} / \mathrm{L}$ & $25.1 \pm 5.4$ & $25.6 \pm 4.8$ & $24.3 \pm 6.1$ & 0.1430 \\
\hline $\mathrm{PaO}_{2} / \mathrm{FiO}_{2}, \mathrm{~mm} \mathrm{Hg}$ & $\begin{array}{c}141.1 \\
{[115.6-188.9]}\end{array}$ & $\begin{array}{c}151.2 \\
{[122.8-199.2]}\end{array}$ & $\begin{array}{c}129.6 \\
{[100.0-163.4]}\end{array}$ & $<0.001$ \\
\hline$<200 \mathrm{~mm} \mathrm{Hg}$ & $174(79.8 \%)$ & 96 (75.0\%) & $78(86.7 \%)$ & 0.025 \\
\hline \multicolumn{5}{|c|}{ Respiratory parameters at HFNC initiation } \\
\hline $\mathrm{SpO}_{2}$ & $86[80-90]$ & 88 [83-92] & $84[75-89]$ & $<0.001$ \\
\hline $\mathrm{SpO}_{2} / \mathrm{FiO}_{2}$ & $\begin{array}{c}108.7 \\
{[91.6-143.3]}\end{array}$ & $\begin{array}{c}121.4 \\
{[100.0-150.0]}\end{array}$ & $\begin{array}{c}97.8 \\
{[85.4-112.5]}\end{array}$ & $<0.001$ \\
\hline $\begin{array}{l}\text { RR (beats per } \\
\text { minute) }\end{array}$ & $25[22-30]$ & $24[22-30]$ & $26[22-30]$ & 0.1319 \\
\hline ROX index & $4.3[3.4-5.5]$ & $4.9[3.8-6.1]$ & $3.8[3.0-4.7]$ & 0.0001 \\
\hline \multicolumn{5}{|c|}{ After HFNC treatment } \\
\hline $\mathrm{SpO}_{2}$ & 93 [90-96] & $95[92-96]$ & $92[89-94]$ & $<0.001$ \\
\hline $\mathrm{SpO}_{2} / \mathrm{FiO}_{2}$ & $\begin{array}{c}117.5 \\
{[105.0-141.0]}\end{array}$ & $\begin{array}{c}134.2 \\
{[117.5-158.3]}\end{array}$ & $\begin{array}{c}108.7 \\
{[94.6-116.3]}\end{array}$ & $<0.001$ \\
\hline $\begin{array}{l}\mathrm{RR} \text { (beats per } \\
\text { minute) }\end{array}$ & $22[21-24]$ & $22[20-24]$ & 23 [22-25] & $<0.001$ \\
\hline Flow (L/min) & $\begin{array}{c}55.0 \\
{[50.0-60.0]}\end{array}$ & $\begin{array}{c}50.0 \\
{[50.0-60.0]}\end{array}$ & $60.0[50.0-60.0]$ & 0.0005 \\
\hline ROX index & $5.2[4.6-6.4]$ & $5.9[5.0-7.5]$ & $4.8[4.1-5.3]$ & $<0.001$ \\
\hline $\begin{array}{l}\text { Length of HFNC, } \\
\text { median [IQR], days }\end{array}$ & $6.0[3.0-11.0]$ & $10.0[7.0-15.0]$ & $3.0[1.0-4.0]$ & $<0.001$ \\
\hline $\begin{array}{l}\text { Median time to MV, } \\
\text { days } \text { II }^{\text {M }}\end{array}$ & $3[1-6]$ & $11[9-18]$ & $2[1-4]$ & $<0.001$ \\
\hline
\end{tabular}

COVID-19, coronavirus disease 2019; Fio 2, fraction of inspired oxygen; IQR, interquartile range; $\mathrm{PaO}_{2}$, partial pressure of oxygen; $\mathrm{PaCO}_{2}$, partial pressure of carbon dioxide. $R O X$, Respiratory rate-oxygenation; SD, standard deviation; Data were expressed median [interquartile range] or as mean \pm standard deviation.

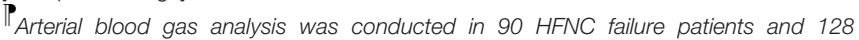
success patients.

IMedian time to MV were available in 141 patients, because do-not-intubate or noninvasive ventilation intolerance was 32 in HFNC failure patients and 23 in success patients.

from the further analysis); $142(60.5 \%)$ patients required MV. The HFNC success probability (84.2\%) for the patients who were divided into low-risk groups following the models was 9.84 times that in the high-risk group (34.8\%) in hospital (Figure 3A).

\section{External Validation of the Models in COVID-19 Patients With HFNC Therapy}

Sixty-nine patients with COVID-19 were included into validation cohort. Baseline characteristics of the patients are shown in Supplementary Tables 1, 2, and there was no significant difference in most characteristics between the two cohorts.

Twenty-six (34.7\%) patients experienced HFNC failure within 7 days of HFNC initiation. The AUC of the novel models is 0.815 (0.70-0.93) (Figure 2B), sensitivity of prediction is $83.8 \%$, and the specificity is $78.1 \%$ in prediction of HFNC failure on the basis of the variables from the scoring system. The HFNC success probability of patients is shown in Figure 3B.

\section{DISCUSSION}

Early identification of COVID-19 patients with AHRF during HFNC therapy at risk of failure will be beneficial for optimal use of medical resources. This retrospective cohort study on COVID19 patients with AHRF showed that age older than 60 years, thrombocytopenia, elevated levels of IL- 6 at HFNC initiation, and an ROX index $<5.31$ within the first $4 \mathrm{~h}$ of HFNC therapy initiation were independent predictors of HFNC failure. The riskstratification model developed for HFNC therapy early predicts the need for MV with greater accuracy than the ROX index alone in COVID-19.

To our knowledge, this report was the largest cohort study to date regarding COVID-19 patients with HFNC therapy. Of patients with analyses on arterial blood gas, the median $\mathrm{PaO}_{2} / \mathrm{FIO}_{2}$ of 141.1 (IQR, 115.6-188.9) indicated the severity of respiratory failure. The HFNC functioned as a bridge between conventional oxygen therapy and $\operatorname{MV}(8,16,19)$. In our cohort, $36.7 \%$ patients with severe AHRF were successfully weaned from HFNC, which provided evidence to the recommendations of a trial of HFNC for COVID-19 patients with moderately severe hypoxemia to avoid the need for $\mathrm{MV}$ from recent $\mathrm{WHO}$ and other guidelines $(7,8,26)$. It is reasonable that COVID-19 patients with less severe hypoxemia are more likely to wean from HFNC. In a study of 17 COVID-19 patients with HFNC in Chongqing, China, compared with 10 HFNC success patients with $\mathrm{PaO}_{2} / \mathrm{FIO}_{2}$ of 209 (IQR, 179-376) $\mathrm{mm} \mathrm{Hg}$, the seven HFNC failure patients had a median $\mathrm{PaO}_{2} / \mathrm{FIO}_{2}$ of 159 (IQR, 137188) $\mathrm{mm} \mathrm{Hg}$ (19). In another study, all the eight COVID19 patients with a baseline mean $\mathrm{PaO}_{2} / \mathrm{FIO}_{2}$ of $259.88 \mathrm{~mm}$ $\mathrm{Hg}$ weaned from HFNC successfully and discharged from the hospital subsequently (27).

The global COVID-19 pandemic has led to an exploding demand for ventilators and medical service worldwide, for which many nations, including China, United States, United Kingdom, and Australia, struggled $(28,29)$. In face of severe shortage of ventilators and health care workers, a trial of HFNC treatment seems plausible, but brings new problems. Some patients may suffer from sudden respiratory arrest and even death during the trial, especially older patients. Our finding of variables derived before, at, and shortly after HFNC initiation predicting its success or failure is very promising to balance the need and the risk associated with the trial.

Tests on peripheral neutrophil count, thrombocyte count, and plasma IL-6 level are commonly conducted. Higher numbers of neutrophils in the peripheral blood correlated with the severity of lung damage (30). In our cohort, patients in the HFNC failure group had significantly higher neutrophil counts than those in the HFNC success group, but no difference in the proportion of neutrophilia was observed between the two groups. Most likely, of these COVID-19 patients with HFNC therapy, the severity of lung damage was comparable. In unselected COVID-19 patients, a low lymphocyte count indicates poor outcome, typically higher 


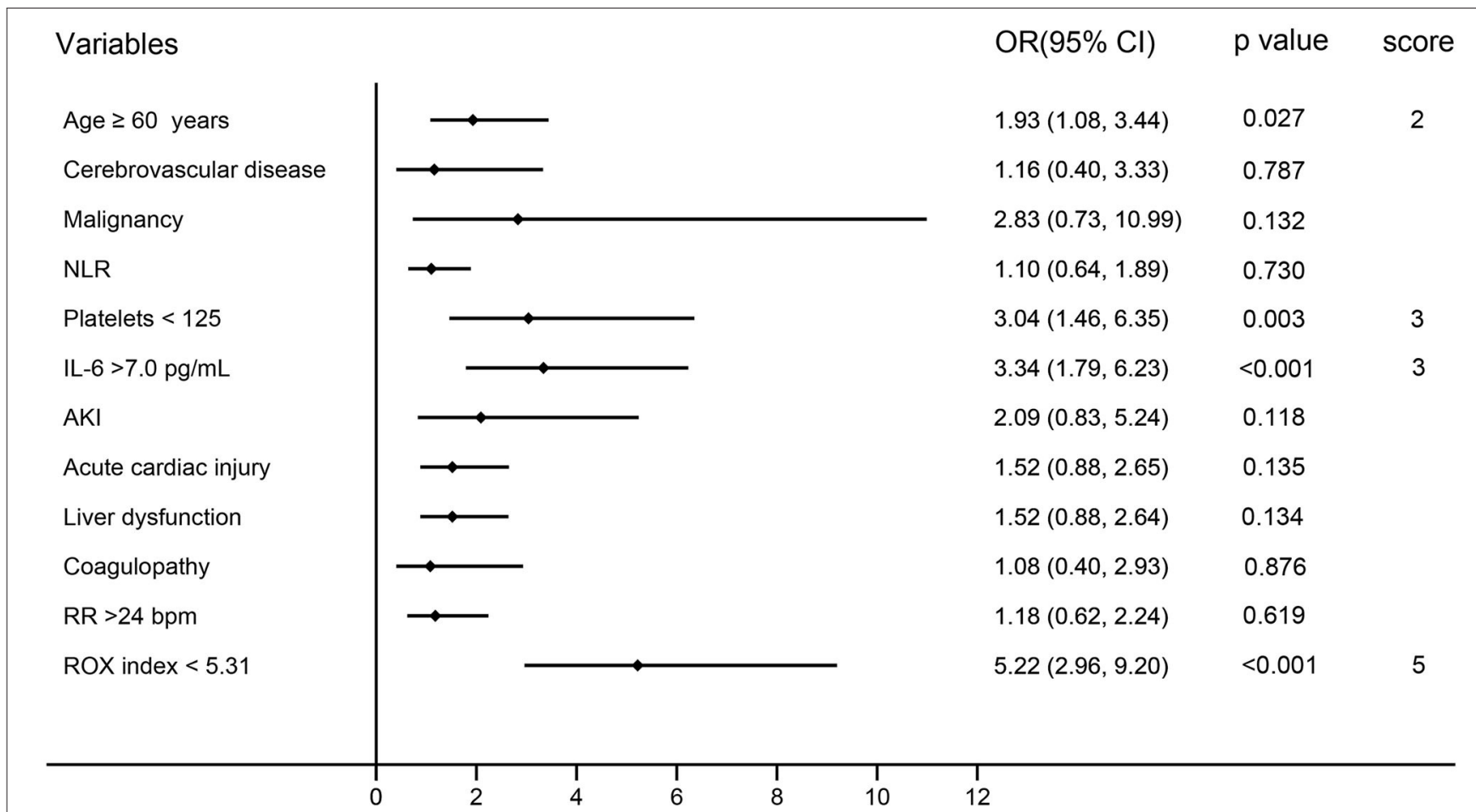

FIGURE 1 | Multivariate analysis and risk-stratification models of HFNC failure in COVID-19 patients. AKI, acute kidney injury; Cl, confidence interval; HFNC, high-flow nasal cannula; IL-6, interleukin 6; NLR, neutrophil-to-lymphocyte ratio; OR, odds ratio; PLT, platelets; ROX, respiratory rate-oxygenation; RR, respiratory rate.

A

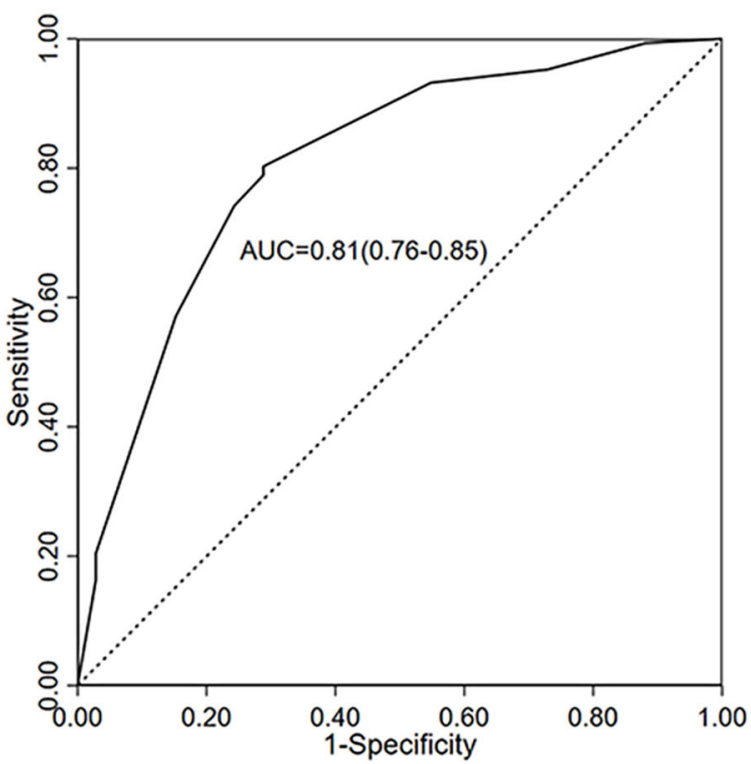

B

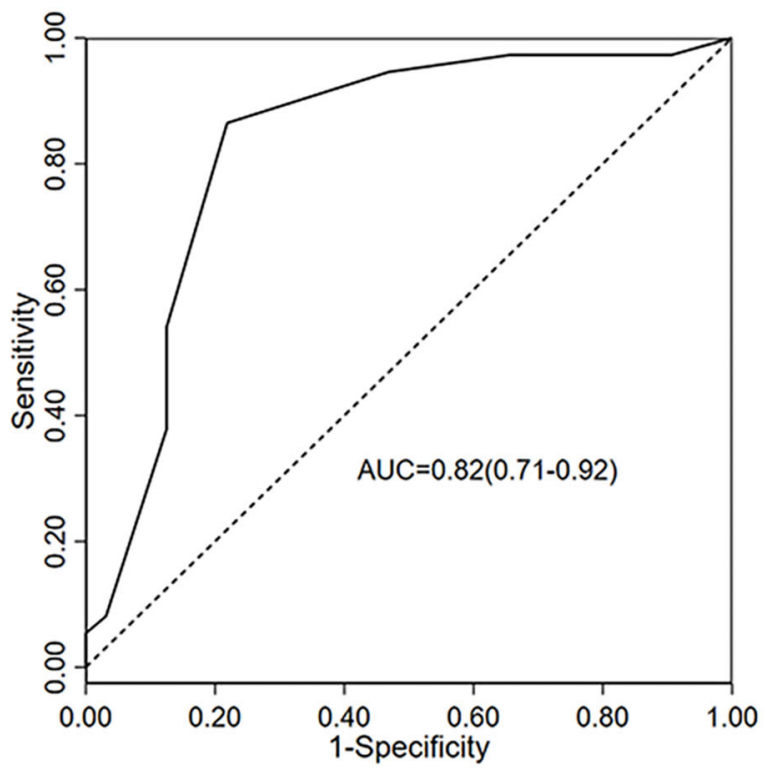

FIGURE 2 | ROC curves for prediction of HFNC failure in COVID-19 patients. (A) AUC of the full regression model in train cohort $(n=324)$; (B) AUC of the Validation cohort $(n=69)$. COVID-19, coronavirus disease 2019; HFNC, high-flow nasal cannula; ROC, receiver operator characteristic; AUC, area under the curve.

mortality. However, the lymphocyte counts were not different between patients in HFNC failure group and in the HFNC success group in our study. The primary reason is that the patients in our study were specifically selected. Only critically ill patients were included, and their median lymphocyte count was $0.6 \times 10^{9} / \mathrm{L}$, which was the same as that in the non-survivors previously 
A

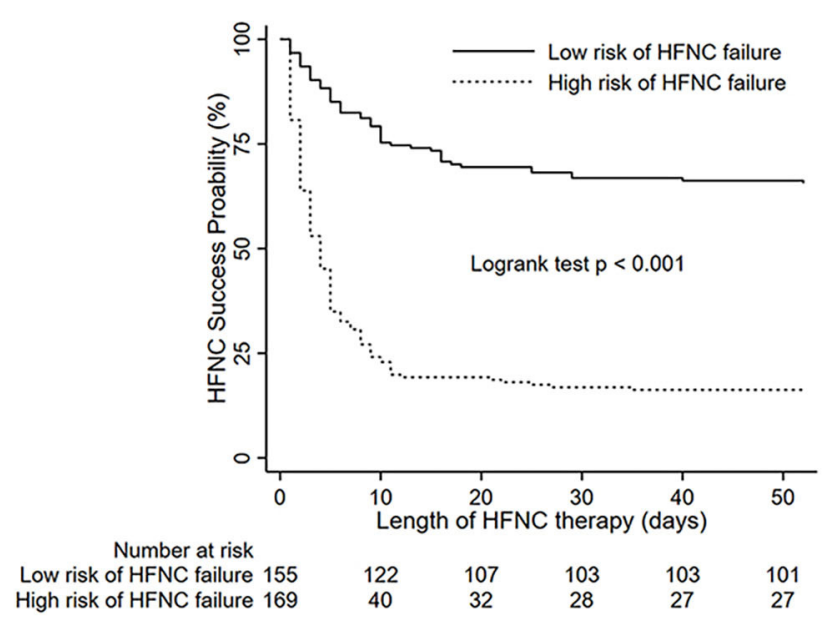

B

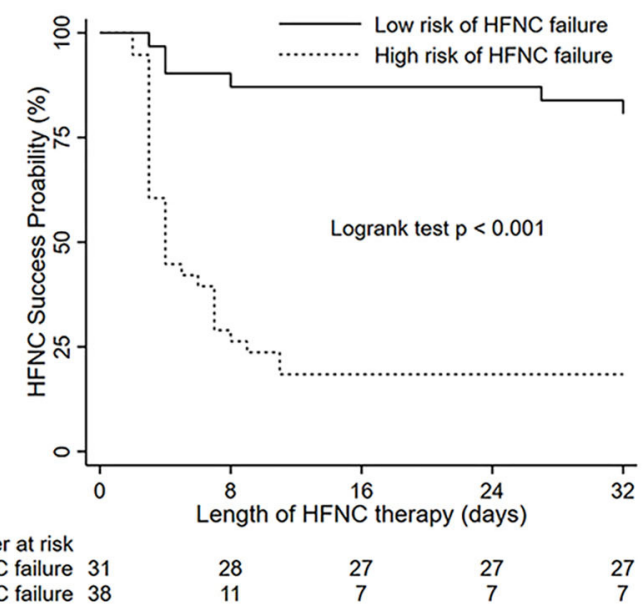

FIGURE 3 | Success curves of HFNC therapy in patients from HFNC onset to termination according to different levels of score. (A) Training cohort; (B) validation cohort; For HFNC failure in hospital: weighted score 0-6 = low risk; $\geq 6=$ high risk. HFNC, high-flow nasal cannula.

reported $(12,13,31)$. To explore the difference in lymphocyte count between the critically ill and the extremely critically ill, a cohort of much larger sample size will be needed. Elevated levels of IL-6, which appears to be from myeloid cells in COVID-19 patients, could predict the severity of the disease and the need for intensive care $(32,33)$. It occurred in $68.8 \%$ of patients in the present study, and the HFNC failure group had more patients with IL-6 above the upper limit of normal range.

ROX index, defined as the ratio of $\mathrm{SpO}_{2} / \mathrm{FIO}_{2}$ to $\mathrm{RR}$, is easily measured at bedside, which has been considered a better predictor of HFNC success compared with $\mathrm{SpO}_{2} / \mathrm{FIO}_{2}$ or RR alone when measured at 2, 6, or $12 \mathrm{~h}$ after HFNC initiation in patients with severe community or hospital-acquired pneumonia $(18,23)$. ROX index $\geq 4.88$ measured within $2-12 \mathrm{~h}$ of HFNC therapy was associated with increased likelihood of HFNC success in non-virus pneumonia $(18,23)$. In our study, COVID19 patients who had an ROX index $\geq 5.31$ within the first $4 \mathrm{~h}$ of HFNC therapy were less likely to need MV.

Advanced age, comorbidities, neutrophilia, and organ and coagulation dysfunction were statistically associated with the development of acute respiratory distress syndrome (ARDS) for COVID-19 patients, which is a determinant of the outcome of HFNC therapy (4); it is thus not reasonable to deny the important of characteristics and clinical parameters. Our novel scoring system covering COVID-19 patients' conditions, physiological variables, and laboratory detection had better predictive capacity in comparison with ROX index alone. Moreover, our scoring system shows promise for the early risk stratification of COVID19 patients with HFNC therapy after appropriate weight.

MV is the rescue therapy for severe AHRF after HFNC failure $(5,7,10,34)$. In our study, among the patients receiving subsequent $\mathrm{MV}, 78.5 \%$ of these patients were deceased. When comparing the COVID-19 patients who received MV before and after $48 \mathrm{~h}$ of HFNC, there are no significant differences in 90-day mortality (Supplementary Figure 1). The proportion of patients who received the MV was different from the HFNC failure rate of patients within 7 days of HFNC onset. We postulated that the primary contributor for the difference was the progressive pathophysiological processes.

During the relatively early stage in patients with severe COVID-19, platelets take part in the formation of pulmonary microthrombi to block the viral invasion into bloodstream when SARS-CoV-2 infects the lung $(35,36)$. The pathophysiological process causes thrombocytopenia and elevation of IL-6, a key proinflammatory cytokine in thromboinflammatory processes (37-39). In general, pulmonary microthrombi lead to shunt in the lung vasculature, but not to decrease in lung volume, which explains the development of atypical ARDS, and so-called silent hypoxemia (40-42).

This study had several limitations. First, during the pandemic of COVD-19, oxygen therapy was the cornerstone of treating severe and critically ill patients, on which it was hard to conduct randomized controlled study. Our study was a retrospective study from a severely stricken place by the disease. The findings were at least informative to physicians from similar locations. Second, some important data, for example, $\mathrm{PaO}_{2} / \mathrm{FIO}_{2}$, were not available for all the patients and were not included in the regression model. However, all the identified predictors were readily accessible, except for places with extremely limited resources. Third, the sample size is not large enough, and we are expecting further studies.

\section{CONCLUSIONS}

Thrombocytopenia, elevated levels of IL-6 at HFNC initiation, and an ROX index $<5.31$ within the first $4 \mathrm{~h}$ of HFNC therapy were independent predictors of HFNC failure, and the riskstratification model on the basis of the four parameters, has a strong predictive ability for the need for MV in COVID-19 patients with HFNC therapy and can further classify the risk 
of HFNC failure. The mortality of HFNC failure patients who received $\mathrm{MV}$ before and after $48 \mathrm{~h}$ of HFNC therapy was not associated with a worse prognosis. A practical oxygen therapy for severe COVID-19 based on our findings may be proposed to prevent from or relieve the overwhelmed health care systems in resource-limited countries, where ICU devices and techniques may not be available or ICU care cannot be provided.

\section{DATA AVAILABILITY STATEMENT}

The original contributions presented in the study are included in the article/Supplementary Material. Further inquiries can be directed to the corresponding author/s.

\section{ETHICS STATEMENT}

The studies involving human participants were reviewed and approved by Wuhan Union Hospital (2020-0041-1). Written informed consent for participation was not required for this study in accordance with the national legislation and the institutional requirements.

\section{AUTHOR CONTRIBUTIONS}

JX, XY, CH, XZo, TZ, SP, LY, YWu, YO, YWa, HQ, and SF collected the epidemiological and clinical data. JX, XY, XZh, WX, DX, LY, and LR summarized all data. JX, XY, LY, YY, HL, YS, and

\section{REFERENCES}

1. Berlin DA, Gulick RM, Martinez FJ. Severe covid-19. $N$ Engl J Med. (2020). doi: 10.1056/NEJMcp2009575. [Epub ahead of print].

2. Huang C, Wang Y, Li X, Ren L, Zhao J, Hu Y, et al. Clinical features of patients infected with 2019 novel coronavirus in Wuhan, China. Lancet. (2020) 395:497-506. doi: 10.1016/S0140-6736(20)30183-5

3. Wang D, Hu B, Hu C, Zhu F, Liu X, Zhang J, et al. Clinical characteristics of 138 hospitalized patients with 2019 novel coronavirus-infected Pneumonia in Wuhan, China. JAMA. (2020) 323:1061-9. doi: 10.1001/jama.2020.1585

4. Wu C, Chen X, Cai Y, Xia J, Zhou X, Xu S, et al. Risk factors associated with acute respiratory distress syndrome and death in patients with coronavirus disease 2019 Pneumonia in Wuhan, China. JAMA Intern Med. (2020) 180:111. doi: 10.1001/jamainternmed.2020.0994

5. Frat JP, Thille AW, Mercat A, Girault C, Ragot S, Perbet S, et al. High-flow oxygen through nasal cannula in acute hypoxemic respiratory failure. $N$ Engl J Med. (2015) 372:2185-96. doi: 10.1056/NEJMoa1503326

6. Matthay MA, Aldrich JM, Gotts JE. Treatment for severe acute respiratory distress syndrome from COVID-19. Lancet Respir Med. (2020) 8:4334. doi: 10.1016/S2213-2600(20)30127-2

7. Alhazzani W, Møller MH, Arabi YM, Loeb M, Gong MN, Fan E, et al. Surviving sepsis campaign: guidelines on the management of critically ill adults with coronavirus disease 2019 (COVID-19). Intensive Care Med. (2020) 46:854-87. doi: 10.1097/CCM.0000000000004363

8. McEnery T, Gough C, Costello RW. COVID-19: respiratory support outside the intensive care unit. Lancet Respir Med. (2020) 8:5389. doi: 10.1016/S2213-2600(20)30176-4

9. Li J, Fink JB, Ehrmann S. High-flow nasal cannula for COVID19 patients: low risk of bio-aerosol dispersion. Eur Respir J. (2020) 55:2000892. doi: 10.1183/13993003.00892-2020

10. Kang BJ, Koh Y, Lim CM, Huh JW, Baek S, Han M, et al. Failure of high-flow nasal cannula therapy may delay intubation and increase mortality. Intensive Care Med. (2015) 41:623-32. doi: 10.1007/s00134-015-3693-5
SF contributed to literature search and drafted the manuscript. DC, SY, DZ, and YS designed the study, have full access to all the data and take full responsibility for accuracy of the analyses and their interpretation. All authors contributed to the article and approved the submitted version.

\section{FUNDING}

This study was supported by grants from the National Key Research and Development Project (2020YFC0841300, 2020YFC0843700, and 2020YFC0843900), and the Major Projects of National Science and Technology on New Drug Creation and Development (2020ZX09201001).

\section{ACKNOWLEDGMENTS}

We thank all HCWs and their families involved in the study. We thank Yuwen Li, MD, Ph.D., Assistant Professor of Pediatrics and Assistant Director of Molecular Genetics and Cytogenetics, Hayward Genetics Center, Tulane University School of Medicine, for language editing and valuable suggestions.

\section{SUPPLEMENTARY MATERIAL}

The Supplementary Material for this article can be found online at: https://www.frontiersin.org/articles/10.3389/fmed. 2020.607821/full\#supplementary-material

11. Chinese management guideline for COVID-19 (version 6.0). Chinese Management Guideline for COVID-19 (version 6.0). (2020). Available online at: http://www.nhc.gov.cn/yzygj/s7653p/202003/46c9294a7dfe4ce f80dc7f5912eb1989/files/ce3e6945832a438eaae415350a8ce964.pdf (accessed October 24, 2020).

12. Yang X, Yu Y, Xu J, Shu H, Xia J, Liu H, et al. Clinical course and outcomes of critically ill patients with SARS-CoV-2 pneumonia in Wuhan, China: a single-centered, retrospective, observational study. Lancet Respir Med. (2020) 8:475-81. doi: 10.1016/S2213-2600(20)30079-5

13. Zhou F, Yu T, Du R, Fan G, Liu Y, Liu Z, et al. Clinical course and risk factors for mortality of adult inpatients with COVID-19 in Wuhan, China: a retrospective cohort study. Lancet. (2020) 395:105462. doi: 10.1016/S0140-6736(20)30566-3

14. Azoulay E, Lemiale V, Mokart D, Nseir S, Argaud L, Pène F, et al. Effect of high-flow nasal oxygen vs standard oxygen on 28-day mortality in immunocompromised patients with acute respiratory failure: the high randomized clinical trial. JAMA. (2018) 320:2099-107. doi: 10.1001/jama.2018.14282

15. Kluge S, Janssens U, Welte T, Weber-Carstens S, Marx G, Karagiannidis C. German recommendations for critically ill patients with COVID-19. Med Klin Intensivmed Notfmed. (2020) p. 1-4. doi: 10.1007/s00063-02000689-w

16. Stéphan F, Barrucand B, Petit P, Rézaiguia-Delclaux S, Médard A, Delannoy $B$, et al. High-flow nasal oxygen vs noninvasive positive airway pressure in hypoxemic patients after cardiothoracic surgery: a randomized clinical trial. JAMA. (2015) 313:2331-9. doi: 10.1001/jama.2015.5213

17. Yu Y, Xu D, Fu S, Zhang J, Yang X, Xu L, et al. Patients with COVID19 in 19 ICUs in Wuhan, China: a cross-sectional study. Crit Care. (2020) 24:219. doi: 10.1186/s13054-020-02939-x

18. Roca O, Caralt B, Messika J, Samper M, Sztrymf B, Hernández G, et al. An index combining respiratory rate and oxygenation to predict outcome of nasal high-flow therapy. Am J Respir Crit Care Med. (2019) 199:136876. doi: 10.1164/rccm.201803-0589OC 
19. Wang K, Zhao W, Li J, Shu W, Duan J. The experience of high-flow nasal cannula in hospitalized patients with 2019 novel coronavirus-infected pneumonia in two hospitals of Chongqing, China. Ann Intensive Care. (2020) 10:37. doi: 10.1186/s13613-020-00653-Z

20. Khwaja A. KDIGO clinical practice guidelines for acute kidney injury. Nephron Clin Pract. (2012) 120:c179-184. doi: 10.1159/000339789

21. Zhang C, Shi L, Wang FS. Liver injury in COVID-19: management and challenges. Lancet Gastroenterol Hepatol. (2020) 5:42830. doi: 10.1016/S2468-1253(20)30057-1

22. Singer M, Deutschman CS, Seymour CW, Shankar-Hari M, Annane D, Bauer $\mathrm{M}$, et al. The third international consensus definitions for sepsis and septic shock (Sepsis-3). JAMA. (2016) 315:801-10. doi: 10.1001/jama.2016.0287

23. Roca O, Messika J, Caralt B, García-de-Acilu M, Sztrymf B, Ricard JD, et al. Predicting success of high-flow nasal cannula in pneumonia patients with hypoxemic respiratory failure: the utility of the ROX index. J Crit Care. (2016) 35:200-5. doi: 10.1016/j.jcrc.2016.05.022

24. Blez D, Soulier A, Bonnet F, Gayat E, Garnier M. Monitoring of highflow nasal cannula for SARS-CoV-2 severe pneumonia: less is more, better look at respiratory rate. Intensive Care Med. (2020) 46:2094209. doi: 10.1007/s00134-020-06199-9

25. Verity R, Okell LC, Dorigatti I, Winskill P, Whittaker C, Imai N, et al. Estimates of the severity of coronavirus disease 2019: a model-based analysis. Lancet Infect Dis. (2020) 20:669-77. doi: 10.1016/S1473-3099(20)30243-7

26. WHO. Infection Prevention and Control During Health Care When Novel Coronavirus (Ncov) Infection is Suspected: Interim Guidance. (2020). Available online at: https://apps.who.int/iris/rest/bitstreams/1266296/retrieve (accessed October 24, 2020).

27. Geng S, Mei Q, Zhu C, Yang T, Yang Y, Fang X, et al. High flow nasal cannula is a good treatment option for COVID-19. Heart Lung. (2020) 49:444-5. doi: 10.1016/j.hrtlng.2020.03.018

28. Clarke AL, Stephens AF, Liao S, Byrne TJ, Gregory SD. Coping with COVID-19: ventilator splitting with differential driving pressures using standard hospital equipment. Anaesthesia. (2020) 75:872-80. doi: 10.1111/ana e.15078

29. Klein A. Who will get ventilators in a covid-19 crisis? New Sci. (2020) 245:12. doi: 10.1016/S0262-4079(20)30663-1

30. Wang J, Li Q, Yin Y, Zhang Y, Cao Y, Lin X, et al. Excessive neutrophils and neutrophil extracellular traps in COVID-19. Front Immunol. (2020) 11:2063. doi: 10.3389/fimmu.2020.02063

31. Xu J, Yang X, Yang L, Zou X, Wang Y, Wu Y, et al. Clinical course and predictors of 60-day mortality in 239 critically ill patients with COVID19: a multicenter retrospective study from Wuhan, China. Crit Care. (2020) 24:394. doi: 10.1186/s13054-020-03098-9

32. Gubernatorova EO, Gorshkova EA, Polinova AI, Drutskaya MS. IL-6: relevance for immunopathology of SARS-CoV-2. Cytokine Growth Factor Rev. (2020) 53:13-24. doi: 10.1016/j.cytogfr.2020.05.009
33. Liao M, Liu Y, Yuan J, Wen Y, Xu G, Zhao J, et al. Single-cell landscape of bronchoalveolar immune cells in patients with COVID-19. Nat Med. (2020) 26:842-4. doi: 10.1038/s41591-020-0901-9

34. Frat JP, Ragot S, Girault C, Perbet S, Prat G, Boulain T, et al. Effect of noninvasive oxygenation strategies in immunocompromised patients with severe acute respiratory failure: a post-hoc analysis of a randomised trial. Lancet Respir Med. (2016) 4:646-52. doi: 10.1016/S2213-2600(16)30093-5

35. Glas GJ, van Der Sluijs KF, Schultz MJ, Hofstra JJ, van Der Poll T, Levi $\mathrm{M}$. Bronchoalveolar hemostasis in lung injury and acute respiratory distress syndrome. J Thromb Haemost. (2013) 11:17-25. doi: 10.1111/jth.12047

36. Yang X, Yang Q, Wang Y, Wu Y, Xu J, Yu Y, et al. Thrombocytopenia and its association with mortality in patients with COVID-19. J Thromb Haemost. (2020) 75:872-80. doi: 10.1111/jth.14848

37. Becker RC. COVID-19 update: covid-19-associated coagulopathy. J Thromb Thrombolysis. (2020) 50:54-67. doi: 10.1007/s11239-020-02134-3

38. McGonagle D, O'Donnell JS, Sharif K, Emery P, Bridgewood C. Immune mechanisms of pulmonary intravascular coagulopathy in COVID-19 pneumonia. Lancet Rheumatol. (2020) 2:E43745. doi: 10.1016/S2665-9913(20)30121-1

39. Ranucci M, Ballotta A, Di Dedda U, Bayshnikova E, Dei Poli M, Resta M, et al. The procoagulant pattern of patients with COVID-19 acute respiratory distress syndrome. J Thromb Haemost. (2020) 18:174751. doi: $10.1111 /$ jth. 14854

40. Gattinoni L, Coppola S, Cressoni M, Busana M, Rossi S, Chiumello D. COVID-19 does not lead to a "typical" acute respiratory distress syndrome. Am J Respir Crit Care Med. (2020) 201:1299-300. doi: 10.1164/rccm.202003-0817LE

41. Gattinoni L, Chiumello D, Caironi P, Busana M, Romitti F, Brazzi L, et al. COVID-19 pneumonia: different respiratory treatments for different phenotypes? Intensive Care Med. (2020) 46:1099-102. doi: 10.1007/s00134-020-06033-2

42. Marini JJ, Gattinoni L. Management of COVID-19 respiratory distress. JAMA. (2020) 323:2329-30. doi: 10.1001/jama.2020.6825

Conflict of Interest: The authors declare that the research was conducted in the absence of any commercial or financial relationships that could be construed as a potential conflict of interest.

Copyright (c) 2020 Xu, Yang, Huang, Zou, Zhou, Pan, Yang, Wu, Ouyang, Wang, Xu, Zhao, Shu, Jiang, Xiong, Ren, Liu, Yuan, Qi, Fu, Chen, Zhang, Yuan and Shang. This is an open-access article distributed under the terms of the Creative Commons Attribution License (CC BY). The use, distribution or reproduction in other forums is permitted, provided the original author(s) and the copyright owner(s) are credited and that the original publication in this journal is cited, in accordance with accepted academic practice. No use, distribution or reproduction is permitted which does not comply with these terms. 